\title{
Development of Necrotizing Myopathy Following Interstitial Lung Disease with Anti-signal Recognition Particle Antibody
}

\author{
Tatsuya Kusumoto ${ }^{1}$, Satoshi Okamori ${ }^{1}$, Keita Masuzawa ${ }^{1}$, Takanori Asakura ${ }^{1}$, \\ Naoshi Nishina ${ }^{2}$, Shotaro Chubachi ${ }^{1}$, Katsuhiko Naoki ${ }^{1}$, \\ Koichi Fukunaga $^{1}$ and Tomoko Betsuyaku ${ }^{1}$
}

\begin{abstract}
:
A 72-year-old man was admitted due to dyspnea on exertion with interstitial shadows and elevated serum creatinine kinase (CK). Despite a close examination, which included magnetic resonance imaging (MRI), we could not diagnose myopathy. Prednisolone was administered and gradually tapered. One year later, antisignal recognition particle (SRP) antibody was confirmed and he was re-admitted for hypoxemia with elevated CK. MRI revealed muscle edema and a histopathological examination of a muscle biopsy specimen showed necrotizing myopathy. Prednisolone, cyclosporine, and intravenous immunoglobulin were administered. Physicians should carefully monitor muscle symptoms and serum CK levels in cases of interstitial lung disease with anti-SRP antibodies.
\end{abstract}

Key words: anti-signal recognition particle antibody, anti-SRP antibody, ILD, interstitial lung disease, necrotizing myopathy

(Intern Med 57: 2045-2049, 2018)

(DOI: 10.2169/internalmedicine.0303-17)

\section{Introduction}

Signal recognition particle (SRP) is a cytoplasmic ribonucleoprotein consisting of six polypeptide chains of 72,68 , $54,19,14$, and $9 \mathrm{kDa}$, and one molecule of 7SL RNA (1). Anti-SRP antibodies were first reported by Reeves et al. (2), and are reportedly observed in $4.9-20.0 \%$ of inflammatory myopathy cases (3-6). Anti-SRP antibody-associated inflammatory myopathy, which usually shows histopathological evidence of necrotizing myopathy (6), is often reported to be refractory to glucocorticoid therapy $(2,7)$. In addition to muscle, inflammatory myopathy also involves the skin, joints, and the lungs in patients with anti-SRP antibody $(5,8)$.

The lungs are commonly involved in systemic connective tissue disease (CTD); the most common lung manifestations are interstitial lung disease (ILD) complicated with rheumatoid arthritis, systemic sclerosis, systemic lupus erythemato- sus, polymyositis or dermatomyositis, Sjögren's syndrome, and mixed CTD (9). While it is well known that ILD often develops as one of the initial symptoms in CTD (9), the ILD that develops in association with CTD and idiopathic interstitial pneumonias differs with regard to its response to immunosuppressive therapy (10). Additionally, specific autoantibodies at the time of the diagnosis of ILD are sometimes useful for the diagnosis of CTD-ILD $(11,12)$. Thus, investigations including examinations for symptoms and signs, and serological tests are clinically important and a diagnostic challenge for physicians.

Some anti-SRP antibody-positive patients show pulmonary involvement in the form of ILD. However, little is known about the frequency, clinical characteristics, or the pattern of the onset of ILD in such patients. We herein report a case in which necrotizing myopathy developed during the follow-up of ILD in an anti-SRP antibody-positive patient.

\footnotetext{
${ }^{1}$ Division of Pulmonary Medicine, Department of Medicine, Keio University School of Medicine, Japan and ${ }^{2}$ Division of Rheumatology, Department of Medicine, Keio University School of Medicine, Japan

Received: October 3, 2017; Accepted: November 14, 2017; Advance Publication by J-STAGE: February 28, 2018

Correspondence to Dr. Shotaro Chubachi, bachibachi472000@live.jp
} 


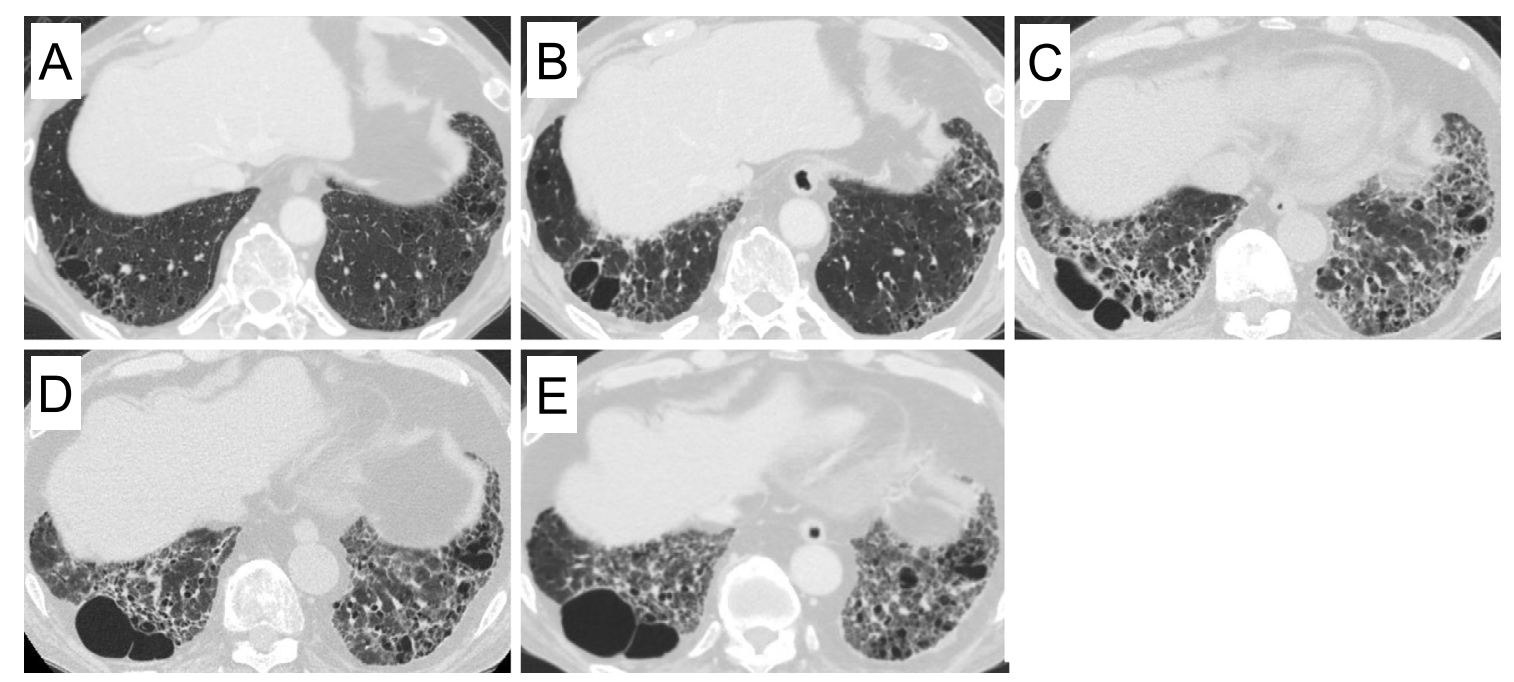

Figure 1. Chest computed tomography 20 months before admission (A), 10 months before admission (B), and at the time of first admission (C) showed the progression of ground glass opacity and reticular shadows. Chest computed tomography at the time of second admission (D) and 3 months after second admission (E).

\section{Case Report}

A 72-year-old man was admitted to our hospital due to dyspnea on exertion (DOE) with progressive interstitial shadows and creatinine kinase (CK) elevation. He had a 17year history of non-small cell lung cancer after treatment with chemoradiotherapy and was under observation for combined pulmonary fibrosis and emphysema. Three-years before the current admission, he had been diagnosed with recurrent lung cancer and was treated with carboplatin plus pemetrexed, followed by pemetrexed maintenance therapy. Ten months before the current admission, ground glass opacities (GGOs) and reticular shadows in the bilateral lower lobes appeared on chest computed tomography (CT), which had not been present on chest CT images obtained 20 months previously (Fig. 1A and B). Pemetrexed was discontinued due to the suspicion of drug-induced pneumonitis; however, the CT findings did not improve. DOE gradually developed over 4 months, with serum CK elevation.

On admission, his oxygen saturation was $93 \%$ while breathing ambient air. His body weight had decreased from $83.4 \mathrm{~kg}$ (10 months before admission) to $80.2 \mathrm{~kg}$. Fine crackles were detectable in the dorsal lung fields, and nail fold bleeding was observed on the right index finger. He had no other signs or symptoms indicating CTD (i.e., joint pain or swelling, proximal muscle weakness, or typical rash of dermatomyositis). Chest CT revealed the progression of the GGOs and reticular shadows in the bilateral lungs (Fig. 1C). A pulmonary function test revealed a decrease in the forced vital capacity (FVC) $[3.39 \mathrm{~L}(91.4 \%$ of predicted)], in comparison to a test that had been performed 20 months previously [4.29 L (113.5\% of predicted)]. Laboratory tests revealed elevated serum CK $[2,191 \mathrm{U} / \mathrm{L}$ (normal: 60-250)], aldolase (ALD) [19.4 IU/L (normal: 2.7-5.9)],
Krebs von den Lungen (KL)-6 [1,367 U/mL (normal: 0500)], and surfactant protein D (SP-D) $[193 \mathrm{ng} / \mathrm{mL}$ (normal: 0 -109)]. A cytoplasmic pattern of anti-nuclear antibodypositivity $(<1: 40)$ was observed, while enzyme-linked immunosorbent assays (ELISAs) were negative for antisynthetase antibody (MBL, Nagoya, Japan), anti-SS-A/Ro antibody, and anti-SS-B/La antibody. We suspected myositisassociated ILD due to the elevated CK level and nail fold bleeding. Among the myositis-specific autoantibodies, antisynthetase antibody and anti-SRP antibody are known to show a cytoplasmic pattern on anti-nuclear antibody tests (13), and among the 6 specific anti-synthetase antibodies (Jo-1, PL-7, PL-12, EJ, KS, and OJ) the ELISA for antisynthetase antibodies cannot detect anti-OJ antibodies. Thus, the presence of anti-OJ or anti-SRP antibodies was suspected in the present case. An RNA immunoprecipitation assay was positive for anti-SRP antibodies. Anti-OJ antibodies are a subtype of anti-synthetase antibodies and are reported to be more closely associated with ILD than myositis or Raynaud's phenomenon (14). However, contrast-enhanced magnetic resonance imaging (MRI) of the lower limbs did not yield evidence of muscle inflammation (Fig. 2A and B). Prednisolone [80 mg/day $(1 \mathrm{mg} / \mathrm{kg})]$ was administered for ILD, and the patient was discharged after the introduction of home oxygen therapy for hypoxemia on exertion. His DOE and elevated serum CK improved, and his prednisolone dose was gradually tapered to $15 \mathrm{mg} / \mathrm{day}$.

One year after being discharged, he was re-admitted to our hospital due to progressive hypoxemia with serum CK elevation. His body weight had decreased to $76.5 \mathrm{~kg}$ over one year. He had no muscle symptoms on admission; however, a physical examination revealed mild muscle weakness in the proximal lower limbs. Chest CT revealed the worsening of GGO and reticular shadows with a peripheral predominantly lower lobe distribution (Fig. 1D). His FVC was 

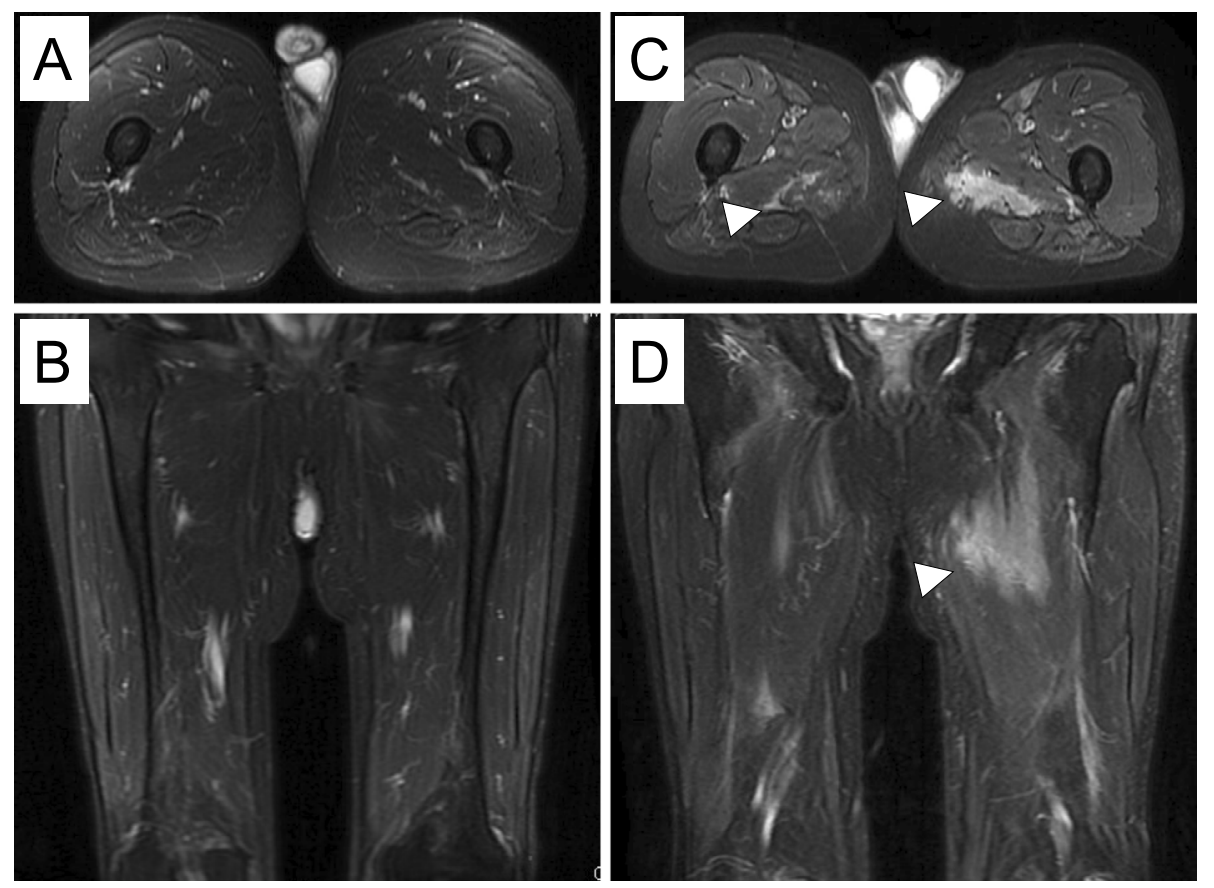

Figure 2. Contrast-enhanced magnetic resonance images (MRI) of the lower limbs at the time of admission (A, B) did not show muscle inflammation. T1-weighted contrast-enhanced MRI axial (C) and coronal (D) images of the lower limbs on re-admission showed hyperintense signals from the internal obturator muscles to the quadriceps, bilaterally (arrowheads).
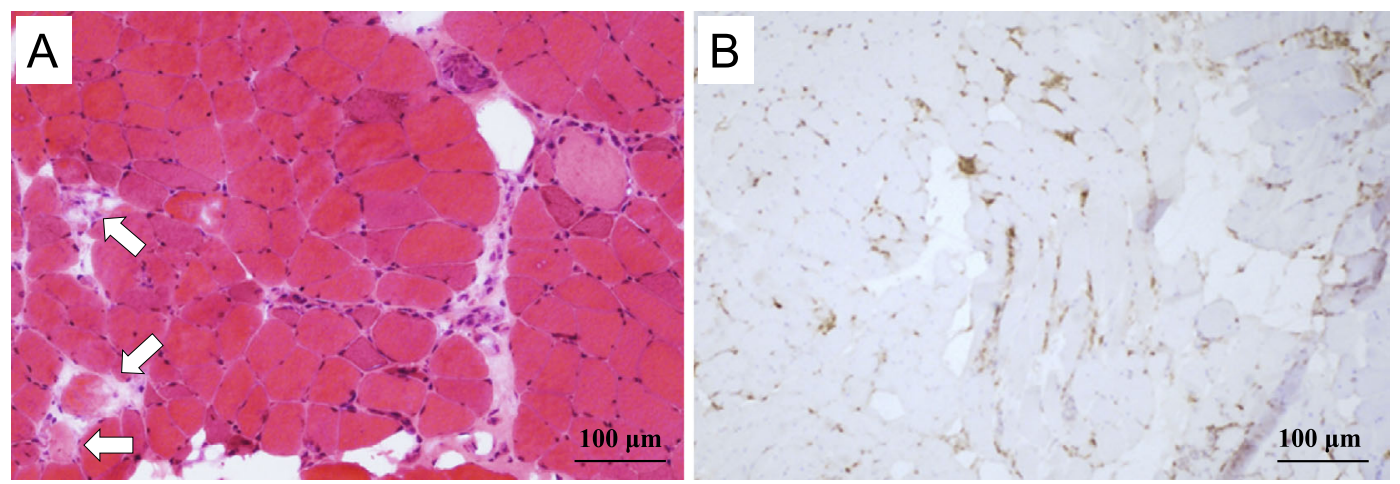

Figure 3. Hematoxylin and Eosin staining of a biopsy of the left quadriceps muscle showed many fibers that were undergoing degeneration (arrows), as well as regenerated fibers; however, the inflammatory cell infiltration was mild (A). Many $\mathrm{CD68}^{+}$cells (macrophages) were present at the sites of the degenerating muscle fibers $(B)$, indicating necrotizing myopathy.

also observed to have decreased from the first admission (3.39 L to $3.27 \mathrm{~L})$. A laboratory analysis revealed elevated serum CK (1,280 U/L), ALD (21.8 IU/L), KL-6 (879 U/ $\mathrm{mL})$, and SP-D (148 ng/mL). Contrast-enhanced MRI of the lower limbs revealed profound muscle edema (Fig. 2C and D). Electromyography indicated myogenic changes accompanied by resting discharge in the gluteus maximus muscle. Quadriceps muscle biopsy revealed numerous necrotic and regenerating fibers, whereas inflammatory cell infiltration around the muscle fibers was slight to mild (Fig. 3); a typical finding in immune-mediated necrotizing myopathy. Aside from typical rash of dermatomyositis he met four of the Bohan and Peter criteria (15).
Treatment with prednisolone (40 mg/day), cyclosporine (150 $\mathrm{mg} /$ day), and intravenous immunoglobulin (IVIG) (30 g/day for 5 days) resulted in CK normalization, but mild muscle weakness, which was considered to be associated with longterm prednisolone treatment, remained. Chest CT and pulmonary function test showed no further progression of ILD (Fig. 1E). The clinical course is shown in Fig. 4.

\section{Discussion}

To clarify the characteristics of ILD in anti-SRP antibodypositive patients, we reviewed the literature in the PubMed database for cases involving anti-SRP antibody-positive pa- 


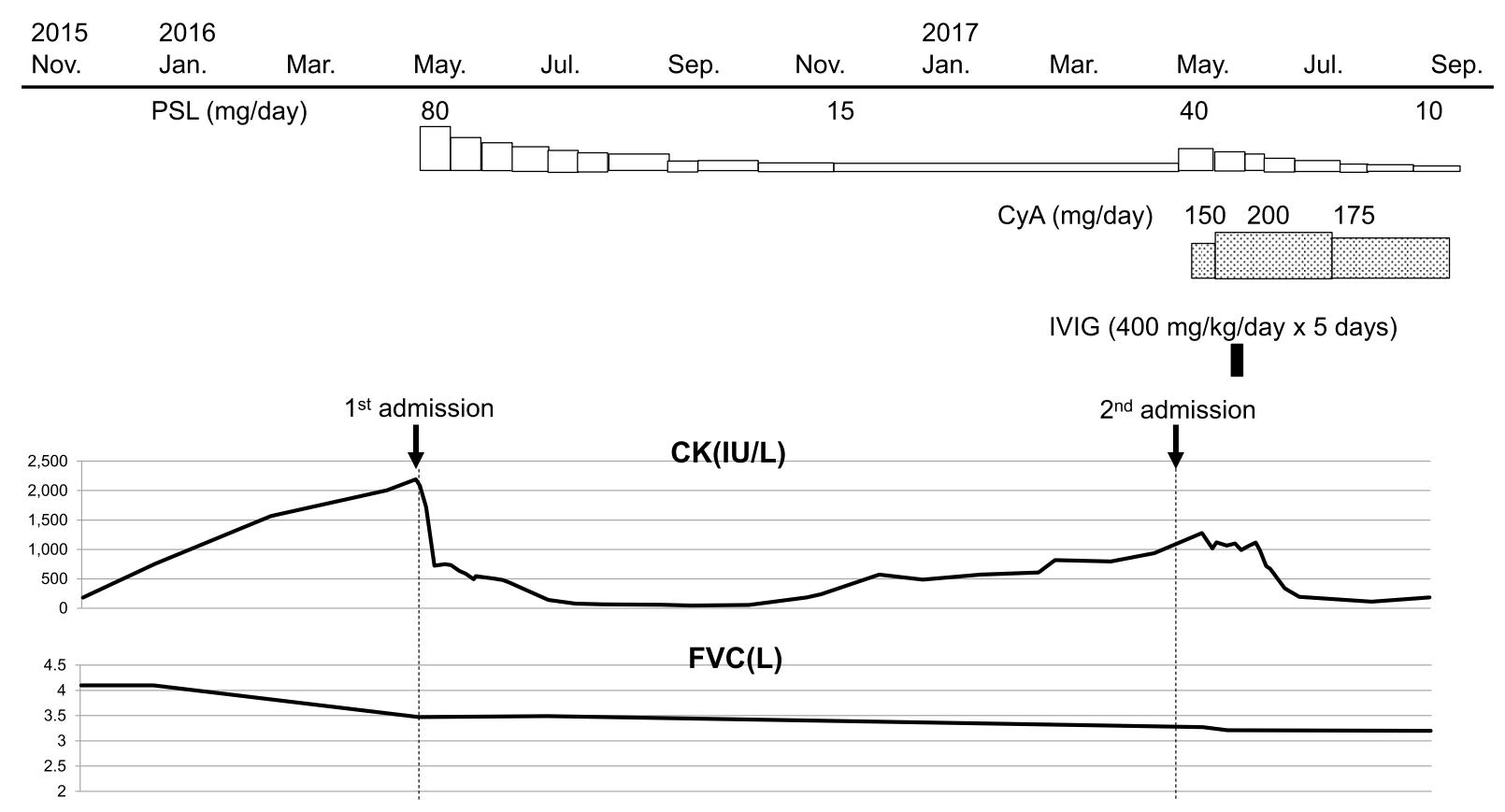

Figure 4. The clinical course of the patient. CK: creatinine kinase, CyA: cyclosporine, FVC: forced vital capacity, IVIG: intravenous immunoglobulin, PSL: prednisolone

tients using the search words "anti-SRP antibody." We identified nine case series involving myopathy-associated antiSRP antibodies (3-5, 16-21), and three case reports of ILD with anti-SRP antibodies (22-24). The nine case series included a total of 239 patients with myopathy-associated anti-SRP antibodies. Forty-one of these 239 (17\%) patients had ILD. With regard to the high-resolution CT pattern of ILD, one study reported that all 13 patients with ILDassociated anti-SRP antibodies showed a non-specific interstitial pneumonia (NSIP) pattern (21). Additionally, the severity of ILD seemed to be mild, and it was reported that the respiratory capacity was reduced due to respiratory or trunk muscle weakness (21). However, no studies on the impact of ILD on the onset or prognosis of myopathy have been reported.

With regard to the three case reports on ILD with antiSRP antibodies (22-24), 5 patients with ILD-associated antiSRP antibodies were identified. Four of these 5 patients had both ILD and myopathy; the remaining patient had ILD alone. Of the 4 patients with ILD and myopathy, 2 patients developed myopathy after ILD $(22,24)$. One patient developed myopathy while being followed without treatment for ILD (NSIP pattern) (24); the other developed myopathy during the tapering of glucocorticoid, which was administered to treat cryptogenic organizing pneumonia (22), which was similar to the current case. However, the patient in that report required intensive treatment, including methyl prednisolone pulse therapy, IVIG, and immunoadsorption to treat progressive muscle weakness and serum CK elevation (>10,000 U/L) (23). In the present case, the early diagnosis of myopathy before the appearance of symptoms facilitated the prompt initiation of therapy and resulted in improvement as we carefully monitored the serum CK levels.
In conclusion, we reported the case of a patient with necrotizing myopathy which developed after ILD with antiSRP antibody-positivity. Physicians should carefully monitor muscle symptoms, signs, and serum CK levels in patients with ILD who are anti-SRP antibody-positive, as an early diagnosis and the prompt initiation of therapy may improve the clinical outcomes of myopathy.

The authors state that they have no Conflict of Interest (COI).

\section{Acknowledgement}

We are grateful to Dr. Matsubara of the Tokyo Metropolitan Neurological Hospital for providing assistance in making the pathological diagnosis, and Professor Kuwana of the Nippon Medical School Graduate School of Medicine for the detection of autoantibodies.

\section{References}

1. Walter P, Blobel G. Purification of a membrane-associated protein complex required for protein translocation across the endoplasmic reticulum. Proc Natl Acad Sci U S A 77: 7112-7116, 1980.

2. Reeves WH, Nigam SK, Blobel G. Human autoantibodies reactive with the signal-recognition particle. Proc Natl Acad Sci U S A 83: 9507-9511, 1986.

3. Targoff IN, Johnson AE, Miller FW. Antibody to signal recognition particle in polymyositis. Arthritis Rheum 33: 1361-1370, 1990.

4. Miller T, Al-Lozi MT, Lopate G, Pestronk A. Myopathy with antibodies to the signal recognition particle: clinical and pathological features. J Neurol Neurosurg Psychiatry 73: 420-428, 2002.

5. Kao AH, Lacomis D, Lucas M, Fertig N, Oddis CV. Anti-signal recognition particle autoantibody in patients with and patients without idiopathic inflammatory myopathy. Arthritis Rheum 50: 209-215, 2004.

6. Suzuki S, Yonekawa T, Kuwana M, et al. Clinical and histological 
findings associated with autoantibodies detected by RNA immunoprecipitation in inflammatory myopathies. J Neuroimmunol 274: 202-208, 2014.

7. Okada N, Mimori T, Mukai R, Kashiwagi H, Hardin JA. Characterization of human autoantibodies that selectively precipitate the 7SL RNA component of the signal recognition particle. J Immunol 138: 3219-3223, 1987.

8. Hoogendijk JE, Amato AA, Lecky BR, et al. 119th ENMC international workshop: trial design in adult idiopathic inflammatory myopathies, with the exception of inclusion body myositis, 10-12 October 2003, Naarden, The Netherlands. Neuromuscul Disord 14: 337-345, 2004.

9. Mathai SC, Danoff SK. Management of interstitial lung disease associated with connective tissue disease. BMJ 352: h6819, 2016.

10. Park JH, Kim DS, Park IN, et al. Prognosis of fibrotic interstitial pneumonia: idiopathic versus collagen vascular disease-related subtypes. Am J Respir Crit Care Med 175: 705-711, 2007.

11. Yoshifuji H, Fujii T, Kobayashi S, et al. Anti-aminoacyl-tRNA synthetase antibodies in clinical course prediction of interstitial lung disease complicated with idiopathic inflammatory myopathies. Autoimmunity 39: 233-241, 2006.

12. Jee AS, Adelstein $S$, Bleasel $J$, et al. Role of autoantibodies in the diagnosis of connective-tissue disease ILD (CTD-ILD) and interstitial pneumonia with autoimmune features (IPAF). J Clin Med 6: 51, 2017.

13. Satoh M, Tanaka S, Ceribelli A, Calise SJ, Chan EK. A comprehensive overview on myositis-specific antibodies: new and old biomarkers in idiopathic inflammatory myopathy. Clin Rev Allergy Immunol 52: 1-19, 2017.

14. Sato S, Kuwana M, Hirakata M. Clinical characteristics of Japanese patients with anti-OJ (anti-isoleucyl-tRNA synthetase) autoantibodies. Rheumatology 46: 842-845, 2007.

15. Bohan A, Peter JB. Polymyositis and dermatomyositis (first of two parts). N Engl J Med 292: 344-347, 1975.

16. Love LA, Leff RL, Fraser DD, et al. A new approach to the classification of idiopathic inflammatory myopathy: myositis-specific autoantibodies define useful homogeneous patient groups. Medicine (Baltimore) 70: 360-374, 1991.

17. Hengstman GJ, ter Laak HJ, Vree Egberts WT, et al. Anti-signal recognition particle autoantibodies: marker of a necrotising myopathy. Ann Rheum Dis 65: 1635-1638, 2006.

18. Takada T, Hirakata M, Suwa A, et al. Clinical and histopathological features of myopathies in Japanese patients with anti-SRP autoantibodies. Mod Rheumatol 19: 156-164, 2009.

19. Suzuki S, Hayashi YK, Kuwana M, Tsuburaya R, Suzuki N, Nishino I. Myopathy associated with antibodies to signal recognition particle: disease progression and neurological outcome. Arch Neurol 69: 728-732, 2012.

20. Hanaoka H, Kaneko Y, Suzuki S, et al. Anti-signal recognition particle antibody in patients without inflammatory myopathy: a survey of 6180 patients with connective tissue diseases. Scand J Rheumatol 45: 36-40, 2016.

21. Suzuki S, Nishikawa A, Kuwana M, et al. Inflammatory myopathy with anti-signal recognition particle antibodies: case series of 100 patients. Orphanet J Rare Dis 10: 61, 2015.

22. Wantke F, Kneussl M, Hubner M, Derfler K, Brücke T, Schmaldienst S. Signal recognition particle (SRP) positive myositis in a patient with cryptogenic organizing pneumonia (COP). Rheumatol Int 30: 1361-1365, 2010.

23. Togawa R, Tanino Y, Nikaido $T$, et al. Three cases of interstitial pneumonia with anti-signal recognition particle antibody. Allergol Int 66: 485-487, 2017

24. Hanaoka H, Kaneko Y, Suzuki S, et al. A unique case of polymyositis with anti-signal recognition particle antibody complicated by subacute interstitial lung disease and subluxing arthropathy, resembling anti-synthetase syndrome. Mod Rheumatol 26: 979-980, 2016.

The Internal Medicine is an Open Access article distributed under the Creative Commons Attribution-NonCommercial-NoDerivatives 4.0 International License. To view the details of this license, please visit (https://creativecommons.org/licenses/ by-nc-nd/4.0/).

(C) 2018 The Japanese Society of Internal Medicine Intern Med 57: 2045-2049, 2018 